\title{
Daigle, France. Sans jamais parler du vent : roman de crainte et d'espoir que la mort arrive à
} temps. Edition critique établie par Monika Boehringer. Moncton : Institut d'études acadiennes, 2012. 259 p.

Monika Boehringer établit dans ces pages une experte édition critique du premier roman de France Daigle, publié une première fois en 1983 et depuis longtemps épuisé. Ce roman expérimental, lumineux de poésie, prose du quotidien à investir de fils d'histoires, explore, selon son auteure dans l'article « De l'avant-texte ou du texte 'dans tous ses états' » paru en 1986 dans La barre du jour et inclus dans le présent ouvrage, "plusieurs niveaux de confusion ». Résolument modernes, ceux-ci lui permettent simultanément de produire un récit détaché de toute spécificité locale, et de reproduire certaines caractéristiques, à savoir le «silence, que côtoie d'ailleurs une langue fort imagée » et un «aspect dénudé de la langue et de l'expression acadienne $»$.

La disposition visuelle du roman renforce cette particularité d'images fortes dans le vide. Notamment, le texte occupe uniquement le tiers inférieur de la page, laissant de larges espaces blancs comme s'ils attendaient une illustration. Peut-être sont-ils une invitation ou une jolie folie littéraire chaque fois qu'un exemplaire du livre trouve une lectrice ou un lecteur. La possibilité d'une telle invitation est d'autant plus envisageable que Sans jamais parler du vent se déroule, en gros (mais pas exclusivement), à l'infinitif; les phrases s'égrènent au rythme de verbes nonconjugués. Les verbes à l'infinitif, ou l'absence de conjugaison, sont fréquemment utilisés pour communiquer les instructions à suivre pour mieux réussir un exercice, et ici la lecture. Par exemple, «Se sentir invincible et doux. Avoir eu besoin de toucher à tout avant d'arriver à cette femme. Caresser une tige de blé en pensant au vent dans ses cheveux. Ne parler que d'elle, ne rêver que d'elle, lui revenir toujours quand les gens se racontent. Situer les choses quelque part dans l'espace et le temps comme si cela existait vraiment l'espace et le temps ». Les personnages, floutés, restent des silhouettes élégantes qui peuplent le roman tout en nous laissant la liberté de les imaginer... ou pas : parmi eux, un homme doux, des femmes, des «ils elles », un androgyne, un voyageur.

Boehringer fournit un appareil critique des plus pertinents. Outre une introduction qui rassemble les motifs et stratégies littéraires privilégiés par Daigle dans ce premier roman, de même que les variantes usuelles d'un pareil projet d'édition, sans oublier l'article de Daigle sur l'avant-texte, Boehringer ajoute de fort intéressantes annexes contenant divers exemples du 
processus d'écriture de Daigle dans le but d'étayer l'analyse stylistique et thématique de la critique. Boehringer porte une attention particulière à « la technique de la narration qui change radicalement d'un version à l'autre ». Au total, sept versions nourrissent la création du roman. Les exemples donnés dans les annexes à la fin du volume éclairent la démarche de Daigle et font apparaitre la profonde altération du projet, au point de transformer du tout au tout les effets et la portée de la narration, ainsi qu'une réduction considérable du texte. Celui-ci devient plus abstrait, plus détaché ; les phrases s'écourtent, nombreuses sont celles qui disparaissent. En exposant la genèse du roman, Boehringer estime que Sans jamais parler du vent forme « un véritable hypertexte ». De là résulte « une couche métatextuelle » qui « relève du système scriptural luimême ». La critique remarque à bon escient qu'à l'époque, la mort de l'auteur a été proclamée et théorisée, et que cet état d'esprit influence Daigle. De la sorte, Daigle a su, comme Nathalie Sarraute dans L'ère du soupçon, procéder à la «déstabilisation, voire l'éclatement, de l'instance énonciative », notamment dans tout ce qui touche au genre et à la sexuation. Boehringer réussit ainsi à dégager, trente ans après la parution du livre, « les principaux motifs, thèmes et structures de base de l'œuvre à venir ». Son édition propose de la sorte un double plaisir de lecture, celle d'un roman fascinant et essentiel dans la carrière littéraire de son auteure, et celle d'un travail critique très soigné, vigoureux et convaincant, qui illumine Sans jamais parler du vent.

Catherine Parayre, Université Brock 